\title{
Inaugural Editorial of Meta-Psychology
}

\author{
Rickard Carlsson, Henrik Danielsson, Moritz Heene, Åse Innes-Ker, Daniël Lakens, Ulrich \\ Schimmack, Felix D. Schönbrodt, Marcel van Assen, Yana Weinstein
}

In 1957 Robert K. Merton wondered how historians living in 2050 would look back at how the sociology of science developed, and predicted that they would see a 'spacious area of neglect' (Merton, 1957, p. 635). Sixty years later, we might safely make a similar prediction about how future historians will look back at the psychology of science. Science is a social enterprise, and psychologists are ideally suited to study the interand intra-individual processes that impact how science is done. One specific area within the psychology of science is the psychology of psychological science, and we refer to this as meta-psychology.

The past several years has seen increased focus on analyzing the systemic and psychological factors that threatens the validity of research in general, and psychology in particular. This focus has resulted in some practical changes, such as journals offering preregistration of hypotheses, platforms for sharing datasets, and an increased discussion on how to improve all aspects of the research cycle from planning to peerreview. This is still work in progress. There are questions about how to increase transparency, how to critique published findings, and how we aggregate results to build a cumulative science. The issues identified can be analyzed from a psychological perspective. For example, we prefer results that confirm our biases, believe prestigious individuals, and remember things selectively. Any recommendations for change should be grounded in an understanding of human psychology in order to work with it, rather than chafing against it.

We believe that it is time to create a specialized jour-

Affiliations: Rickard Carlsson, Linnaeus University, Sweden; Henrik Danielsson, Linköping University, Sweden and The Swedish Institute for Disability Research, Sweden; Moritz Heene, Ludwig-Maximilians-University Munich, Germany; Åse Innes-Ker, Lund University, Sweden; Daniël Lakens, Eindhoven University of Technology, The Netherlands; Ulrich Schimmack, University of Toronto, Canada; Felix D. Schönbrodt, Ludwig-Maximilians-University Munich, Germany; Marcel van Assen, Tilburg University and Utrecht University, The Netherlands; Yana Weinstein, University of Massachusetts, Lowell, USA nal that publishes articles in the emerging field of metapsychological research; a journal that questions the basic assumptions of research paradigms and monitors the progress of psychological science as a whole. The new journal Meta-Psychology aims to provide a platform for academic work on the psychology of psychological science, as well as an outlet for new types of contributions, such as high quality post-publication peer reviews, articles that empty the file-drawers of researchers, and registered reports.

\section{Psychology Needs a Journal Dedicated to Meta-Psychology}

Most scientific journals focus on publishing original research articles or review articles (including metaanalyses) of studies on a particular topic. So far, there has been no outlet dedicated to meta-psychological articles. A large number of high-quality meta-psychological blogs have appeared in recent years, and these blogs are indicative of the need of scholars to communicate ideas, (re)-analyses, and data that do not fit existing scientific outlets. We believe it is time to provide researchers access to a specialized journal that publishes articles in the emerging field of meta-psychology, in order to incorporate this important body of work into the scientific archive, and facilitate open peer-review of these contributions. The journal Meta-Psychology (MP) provides researchers interested in these issues an opportunity to publish their work in a peer-reviewed scientific journal.

Meta-Psychology shares some goals with two journals from the Association for Psychological Science: Perspectives on Psychological Science (PPS) and Advances in Methods and Practices in Psychological Science (AMPPS). In his first editorial (Diener, 2006), Ed Diener described PPS as a journal that publishes an "eclectic mix of provocative reports and articles, including broad integrative reviews, overviews of research programs, meta-analyses, theoretical statements, and articles on topics such as the philosophy of science, opinion pieces about major issues in the field, autobiographical reflections of senior members of the field, and even occasional humorous essays and sketches". AMPPS's website states that it "is the home for innovative developments in research methods, practices, and 
conduct across the full range of areas and topics within psychological science".

We believe that despite the similarities with PPS and AMPPS, Meta-Psychology provides a unique and important outlet for meta-psychological research. Indeed, Meta-Psychology differs from PPS and AMPPS in several important ways. First, PPS and AMPPS are both traditional journals that require readers to pay in order to read the articles. In contrast, Meta-Psychology is an open access journal that is free both for readers (golden open access) and for authors (no article processing fees). Open access is a defining attribute of any journal ascribing to the ideals of openness and transparency. Expensive subscriptions exclude readers who are not in the fortunate position to be employed at a wealthy university. Although Open Access journals solve this problem, they often have expensive article processing charges that will exclude authors who cannot acquire the necessary funding. We firmly believe that in order to ensure diversity in meta-psychological research, the only way forward is completely free open access. We are proud to have made this possible by relying on open source software (Open Journal Systems, https://pkp.sfu.ca/ojs/; the apa6 class for $\mathrm{HT}_{\mathrm{E}} \mathrm{X}$ by Brian Beitzel, 2012), a free infrastructure provided by Linnaeus University, and volunteering editors who are not receiving monetary compensation for their work.

Meta-Psychology will not only be an outlet for examining and advancing psychological science, but also function as an avant garde experiment that pushes the boundaries of scientific publishing. Already at the start, Meta-Psychology will be quite different from traditional scientific journals. Meta-Psychology has no physical or economic limitations in the number of articles that can be published. The sole criterion for publication is that an open peer-review process considers a submission a valuable contribution to the field of meta-psychological research. Manuscripts submitted to Meta-Psychology are first published as pre-prints. The full editorial work is openly available for anyone to inspect, independent on whether an article is ultimately published or rejected. All submissions will be reviewed by two or three expert reviewers selected by the action editor, but anyone can read and comment on pre-prints, and these comments will be incorporated in the formal review process. Commenters who make a substantial contribution will be credited as additional reviewers on a given paper.

In addition to providing an open peer review process and transparent editorial decisions, we also expect high standards of openness from our authors. All published articles have to be accompanied by data, code, and materials posted on an Open Science Framework project page that is cited in the article. The analysis will be independently reproduced by reviewers and/or editors prior to publication. Furthermore, pre-registration is strongly encouraged. In terms of the Transparency and Openness (TOP) guidelines, we aim to reach the highest (level 3) or the next highest level (level 2) on all criteria. For more details see the submission guidelines on our website.

To summarize, Meta-Psychology will have absolutely no barriers with respect to funding and accessibility, a low threshold on perceived novelty and splashy findings, and a high threshold on openness and transparency. Importantly, it is our vision that MetaPsychology will lead the way forward by being an early adopter of advances in how psychological research is conducted and communicated. Indeed, our intention is that Meta-Psychology will be a living and evolving metapsychology experiment in itself.

\section{First Call for Papers}

Meta-Psychology publishes original articles on the topic of meta-psychology, and authors should read the About the Journal section on the website to discover the large scope of submissions the journal will consider. However, when launching we would like to emphasize three types of contributions that we are especially proud to give a home in Meta-Psychology.

\section{File-Drawer Reports}

The overall goal of accepting File-Drawer Reports is to correct the scientific record by encouraging researchers to write up and publish findings that otherwise would remain in the file drawer and bias the literature.

These articles provide an opportunity to completely empty your (or your lab's) file drawer on a psychology research topic. The exhaustiveness of the emptying is crucial, meaning that even suspectedely failed (e.g., manipulation check failed, suspicion of problems with data collection) should be included, but of course carefully detailed and explained in order to give future metaanalysts the information they need to decide whether the data are useful.

File-Drawer Reports should ideally not be rejected papers that would do well as original contributions in other journals. Rather, we literally want you to scrape the bottom of your drawers and submit findings that otherwise would never be published in a scientific journal.

Because this is an entirely new format, there will likely be some initial confusion of what the reports should contain (but please see the submission instructions) and what are appropriate topics. For that reason, we encourage authors to contact Rickard Carlsson 
to discuss ideas about how to go about emptying your file drawer.

\section{Expand your Blog to an Article}

There's no doubt that blogs have played an important role in the emerging field of meta-psychology. Blogs provide a medium to quickly disseminate information that would otherwise be hard to publish in traditional journals. Sometimes blogs are just random musings, but sometimes they contain highly important contributions. We think it would be a shame if these contributions did not become part of the scientific record.

Although you may independently start re-writing your blog into a submission type that fits MetaPsychology (see submission guidelines), we encourage you to contact an editorial board member to discuss whether your blog post has the potential to be expanded to an MP article, before you start working on it.

\section{Registered Reports in Meta-Psychology}

We are proud to have the option to submit proposals for meta-psychological research that will be given inprinciple acceptance (IPA) prior to any data collection and/or data analysis. Start by consulting the submission guidelines, and if you have any more questions you should consult with the Registered Report section editor Marcel van Assen.

\section{Outlook}

Being on the frontier of the ongoing changes in academic publishing means that we are experimental and thus modest in that we might not get everything right from the start. Expect Meta-Psychology to evolve and be the first adopter of important meta-science and publication process advances.

\section{References}

Beitzel, B. (2012). Formatting latex documents in apa style (6th edition) using the apa6 class. The PracTEX Journal, 2012(1), 1-12.

Diener, E. (2006). Editorial. Perspectives on Psychological Science, 1, 1-4. doi:10.1111/j.1745-6916.2006. 00001.x

Merton, R. K. (1957). Priorities in scientific discovery: a chapter in the sociology of science. American Sociological Review, 22, 635-659. doi:10.2307/ 2089193 


\section{Co-Editors-in-Chief (2017 - 2021)}

Information about the journal

- Rickard Carlsson (Linnaeus University, Sweden)

- Ulrich Schimmack (University of Toronto, Mississauga, Canada)

Editorial Board (2017 - 2021)

- Henrik Danielsson (Linköping University, Sweden)

- Moritz Heene (Ludwig-Maximilians-University Munich, Germany)

- Åse Innes-Ker (Lund University, Sweden)

- Daniël Lakens (Eindhoven University of Technology, The Netherlands)

- Felix Schönbrodt (Ludwig-Maximilians-University Munich, Germany)

- Marcel van Assen (Tilburg University, and Utrecht University, The Netherlands)

- Yana Weinstein (University of Massachusetts, Lowell, USA)

Advisory board (2017 - 2021)

- Nick Brown (University of Groningen, The Netherlands)

- Paul Bürkner (University of Münster, Germany)

- Gary Burns (Wright State University, USA)

- Cody Christopherson (Southern Oregon University, USA)

- James Coyne (University of Pennsylvania, USA)

- Malte Elson (Ruhr University Bochum, Germany)

- Eiko Fried (University of Amsterdam, The Netherlands)

- Kristoffer Magnusson (Karolinska Institutet, Sweden)

- Stephen Martin (Baylor University, USA)

- David Meyer (University of Michigan, Ann Arbor, USA)

- Julia Rohrer (University of Leipzig, Germany)

- Anne Scheel (Eindhoven University of Technology, The Netherlands)

- Donald Williams (University of California, Davis, USA)

- Matt Williams (Massey University, New Zealand)

- Tim van der Zee (Leiden University, The Netherlands)

Links

- The journal's website: https://open.lnu.se/index.php/metapsychology

- The journal's OSF project: https://osf.io/3m4z3/ 